\title{
HIV in female sex workers in five border provinces of Vietnam
}

\author{
N V Thuong, V T Nhung, K V Nghia, L T Tram, N O'Farrell
}

Sex Transm Infect 2005;81:477-479. doi: 10.1136/sti.2005.016097

Objectives: To determine the prevalence of HIV and associated risk factors among female sex workers (FSWs) in border provinces of Vietnam.

Methods: 911 FSWs in five border provinces of Vietnam (Lai Chau, Quang Tri, Dong Thap, An Giang, and Kien Giang) were enrolled in a cross sectional study. Subjects were interviewed using a standardised questionnaire about selected sociodemographic and behavioural characteristics, history of STls, and information about their cohabiting partners (husbands or live-in partners). Serological tests were done for HIV and syphilis (TPHA+RPR) and urine tests (PCR) for chlamydia and gonorrhoea. Associations between HIV and selected features of FSWs and their partners were examined using univariate and multivariate logistic regression analysis.

Results: Overall, the prevalence of HIV among FSWs in the five provinces of Vietnam was $4.5 \%$. The prevalence of HIV was higher in the southern border regions $(4.0 \%-7.0 \%)$ than the northern $(2 \%)$ and central $(1 \%)$ regions. In multivariate analysis between HIV and selected features of FSWs, income $\leqslant \$ 33 /$ month (OR 2.36, $p=0.04$ ), age of first sex $\leqslant 15$ $(O R=5.48, p=0.005)$, and $\geqslant 9$ clients per week (OR 2.80, $p=0.018$ ) were associated with HIV infection. Positive syphilis serology achieved a borderline significant association with HIV (OR 2.30, $p=0.095$ ). Having a regular nonpaying partner $(O R=0.35, p=0.060)$ was a borderline protective factor for HIV.

Conclusion: Interventions to limit HIV transmission among FSWs in Vietnam should be implemented early and focus on young poor populations in these border areas.

$\mathrm{N}$ ational HIV serosurveillance data for Vietnam have indicated a steady increase in the prevalence of HIV. This trend has been particularly noticeable among injecting drug users ( $10.1 \%$ in 1996 to $32 \%$ in 2002) and female sex workers (FSWs) $\left(0.6 \%\right.$ in 1994 to $6.6 \%$ in 2002). ${ }^{1}$ These surveillance data also report widespread variation in the HIV seroprevalence among FSWs in the various provinces with high rates in Hai Phong in the north west and Can Tho, Ho Chi Minh City, and An Giang in the south but low levels in other areas.

In many parts of South East Asia, FSWs are at increased risk of HIV and merit targeted HIV prevention activities. Although sex work is officially illegal in Vietnam, many diverse venues exist where sex is exchanged for money. However, FSWs are often reluctant to identify themselves as such to healthcare providers for fear of being detained in reeducation camps. ${ }^{2}$

Mobile sexually active populations are a recognised risk group for HIV and border areas appear to be particularly vulnerable to high rates of STIs and HIV. The Community
Action for Preventing HIV/AIDS project (JFPR-9006) was designed to target selected border provinces in three Mekong countries, Vietnam, Cambodia, and Laos, with an extensive HIV prevention package involving information, education and communication, condom promotion, and improved STI control both in the general population and FSW. ${ }^{3}$ In Vietnam five provinces were selected and this study was undertaken to determine both the prevalence of HIV in FSWs and risk factors for infection in order to inform future HIV prevention interventions.

\section{METHODS}

In all, 911 FSWs in five border provinces-Lai Chau in the north, Quang Tri in the centre, and An Giang, Dong Thap, and Kien Giang in the south participated in a cross sectional study between December 2002 and February 2003, using available mapping information obtained from the project baseline survey. ${ }^{4}$ Informed verbal consent was obtained from all subjects. FSWs who requested HIV test results were referred to the Department of Preventive Medicine for voluntary counselling and testing.

Using a standard interview schedule in Vietnamese, subjects were identified in their place of work and interviewed by a healthcare worker about selected sociodemographic characteristics, sexual behaviour, healthcare seeking behaviour, history of STIs, and selected features regarding cohabiting partners (husbands or live-in partners) as reported elsewhere. ${ }^{56}$ Study subjects were classified into direct female sex workers (DFSWs) selling sex from streets, parks, bus stops, boats, ferry pier, brothels and guesthouses, and indirect female sex workers, working and selling sex in hotels, restaurants, massage parlours, cafés, karaoke lounges, bars, and barbershops. The sample size was assessed assuming a $5 \%$ prevalence of HIV using the estimated numbers of FSWs in each province obtained for the baseline survey ${ }^{4}$ plus $10 \%$.

HIV infection was determined by positive reactions with all of the following: SFD (SFD HIV 1/2 PA, Bio-Rad, 2002, Tokyo, Japan) and two ELISA tests (Murex HIV 1.2.0, Abbott, 2002, Dartford, UK; Genscreen HIV 1/2 V.2, Bio-Rad, 2002, Marnes La Coquette, France). Urine specimens were tested for gonorrhoea and chlamydia by polymerase chain reaction (PCR) (Amplicor, Roche, 2002, Branchburg, USA). Tests for syphilis were done with rapid plasma reagin (RPR) and Treponema pallidum haemagglutination assay (TPHA) both made by Bio-Rad 2002 (Kentford, UK). Positive cases of syphilis were defined by positive reactions in both tests. All tests were unlinked and anonymous.

Data were entered into Epi-info version 6.04d (CDC, USA, 2001). Odds ratios (with 95\% confidence intervals) were used

Abbreviations: DFSWs, direct female sex workers; FSWs, female sex workers; IDU, injecting drug use; PCR, polymerase chain reaction; RPR, rapid plasma regain; STI, sexually transmitted diseases; TPHA

Treponema pallidum haemagglutination assay 
Table 1 Univariate association between HIV and selected sociodemographic, behavioural variables, STI related factors, and selected features of cohabiting partners of FSWs

\begin{tabular}{|c|c|c|c|c|c|}
\hline Characteristics & No & $\%$ & $\begin{array}{r}\text { Prevalence } \\
\text { of HIV (\%) }\end{array}$ & OR $(95 \% \mathrm{Cl})$ & p Value \\
\hline Kinh ethnicity & 899 & 91.1 & 4.4 & $0.87(0.33$ to 2.95$)$ & $0.774^{F}$ \\
\hline Age 14-20 (reference) & 903 & 23.5 & 4.7 & NA & \\
\hline $21-30$ & 903 & 50.4 & 4.0 & $0.83(0.38$ to 1.91$)$ & 0.65 \\
\hline$>30$ & 903 & 26.1 & 5.5 & $1.18(0.50$ to 2.83$)$ & 0.70 \\
\hline Having cohabiting partner & 903 & 26.2 & 6.8 & 1.85 (0.95 to 3.53$)$ & 0.057 \\
\hline Having a non-paying regular partner & 879 & 34.4 & 2.3 & $0.40(0.16$ to 0.89$)$ & 0.027 \\
\hline None/primary school only & 900 & 60.1 & 6.1 & $2.85(1.34$ to 6.65$)$ & 0.006 \\
\hline Income $\leqslant 33$ ( $\$ /$ month) & 820 & 28.4 & 6.9 & $1.99(1.00$ to 3.89$)$ & 0.041 \\
\hline Working duration $\leqslant 6$ months & 872 & 19.7 & 2.3 & $0.48(0.14$ to 1.28$)$ & 0.164 \\
\hline Worked outside Vietnam & 891 & 3.9 & 11.4 & $3.02(0.87$ to 8.49$)$ & $0.062^{F}$ \\
\hline Direct sex work & 903 & 44.9 & 5.2 & $1.31(0.69$ to 2.47$)$ & 0.401 \\
\hline Age of first sex $\leqslant 15$ & 855 & 3.7 & 15.6 & $4.56(1.48$ to 12.11$)$ & $0.009^{F}$ \\
\hline No of clients/week $\geqslant 9$ & 900 & 23.2 & 7.2 & $2.15(1.08$ to 4.16$)$ & 0.021 \\
\hline \multicolumn{6}{|l|}{$100 \%$ condom use in the last month with } \\
\hline Non-regular customer(s) & 884 & 45.1 & 4.3 & $0.89(0.46$ to 1.70$)$ & 0.731 \\
\hline Regular customer(s) & 515 & 34.0 & 4.0 & $0.75(0.29$ to 1.79$)$ & 0.517 \\
\hline Cohabiting partner(s) & 236 & 9.3 & 0.0 & NA & \\
\hline History of STIs & 845 & 21.5 & 7.1 & $1.88(0.92$ to 3.72$)$ & 0.066 \\
\hline NG/CT & 900 & 19.9 & 6.7 & $1.78(0.86$ to 3.53$)$ & 0.101 \\
\hline Positive syphilis serology & 903 & 10.7 & 8.2 & $2.10(0.89$ to 4.57$)$ & $0.071^{\mathrm{F}}$ \\
\hline Vaginal douching in the last month & 894 & 64.1 & 4.5 & $1.04(0.54$ to 2.08$)$ & 0.902 \\
\hline Visited women's clinics & 898 & 33.6 & 5.0 & $1.19(0.61$ to 2.29$)$ & 0.596 \\
\hline \multicolumn{6}{|c|}{ Selected features of FSW' cohabiting partners } \\
\hline Age $<30$ & 248 & 29.0 & 9.7 & $1.99(0.68$ to 5.68$)$ & $0.252^{\mathrm{F}}$ \\
\hline Occupation involving mobility & 247 & 72.9 & 7.2 & $1.66(0.49$ to 7.48$)$ & $0.568^{\mathrm{F}}$ \\
\hline Ever drug use & 220 & 9.5 & 4.8 & $0.66(0.03$ to 4.06$)$ & $1.0^{\mathrm{F}}$ \\
\hline
\end{tabular}

to measure the magnitude of the association between HIV and selected sociodemographic and behavioural factors. In the univariate analysis, $\chi^{2}$, and Fisher's exact tests were used to identify potential candidates for inclusion in the multivariate logistic regression analysis. Multivariate logistic regression analysis was done using Stata version 6.0 (Stata Corporation, TX, USA 1999). The study protocol and questionnaire were reviewed and approved by the AIDS Division, Ministry of Health, the Vietnam Commission for Population, Family and Children, Population and Family Health, and the Pasteur Institute, Ho Chi Minh City.

\section{RESULTS}

The respective prevalences of HIV, 95\% confidence intervals (CI) and number (n) of FSW in the five provinces were, overall $4.5 \%$ (CI $3.3 \%$ to $6.2 \%$ ), $n=903$; Lai Chau $2.0 \%$ (CI $0.2 \%$ to $7.0 \%$ ), $\mathrm{n}=100$; Quang Tri $1 \%$ (CI $0.0 \%$ to $5.4 \%$ ), $\mathrm{n}=101$; Dong Thap $4.7 \%$ (CI $1.9 \%$ to $9.4 \%$ ), $\mathrm{n}=149$; An Giang $7 \%$ (CI $4.4 \%$ to $10.5 \%) n=300$; and Kien Giang $4 \%$ (CI $1.9 \%$ to $7.1 \%), n=253$. Four per cent of FSWs had used opiates, none of whom were HIV positive.

In univariate analysis, the prevalence of HIV was significantly higher in those with low educational attainment, low income ( $\leqslant \$ 33 /$ month), age of first sex $\leqslant 15$, nine clients or more per week, and those without a non-paying regular partner $(\mathrm{p}<0.05)$. The prevalence of HIV was marginally significantly higher in those having a cohabiting partner, ever having worked outside Vietnam, history of STI, and having a positive test for syphilis $(0.05<\mathrm{p}<0.1)$ (table 1 ).

In multivariate analysis (table 2 ), age of first sex $\leqslant 15$, low income $(\leqslant \$ 33 /$ month $)$, and $\geqslant 9$ clients per week were significantly associated with a higher prevalence of HIV than the corresponding reference groups $(p<0.05)$. Positive syphilis serology was marginally associated with a higher prevalence of HIV $(p=0.095)$, while having a non-paying regular partner was marginally associated with a lower prevalence of HIV $(\mathrm{p}=0.06)$.

\section{DISCUSSION}

We found a variation in HIV prevalence between the five provinces. Variation in HIV prevalence has also been noted within a single province, An Giang, where HIV prevalence in FSWs in recent national sentinel surveys ranged between $16 \%$ and $24 \% .^{7}$ Much of this variation in An Giang, a province that shares a border with Cambodia, is thought to be related to both the numbers sampled from detention camps and temporary migration of Vietnamese FSWs both inside Vietnam and from Cambodia-a STI prevalence survey in Cambodia found that one third of direct sex workers had Vietnamese as their first language. ${ }^{8}$

Table 2 Multivariate association between HIV and selected features of FSWs

\begin{tabular}{lll}
\hline Variable & OR $(95 \% \mathrm{Cl})$ & $\mathbf{p}$ Value \\
\hline Income $\leqslant \$ 33 /$ month & $2.36(1.03$ to 5.37$)$ & 0.04 \\
Age of first sex $\leqslant 15$ & $5.48(1.67$ to 18.00$)$ & 0.005 \\
$\geqslant 9$ clients/week & $2.80(1.19$ to 6.59$)$ & 0.018 \\
Having a non-paying regular partner & $0.35(0.12$ to 1.04$)$ & 0.060 \\
Positive syphilis serology & $2.30(0.86$ to 6.12$)$ & 0.095 \\
\hline
\end{tabular}

Variables in the full model: duration of work, income, clients/week, having a cohabiting partner, having a nonpaying regular partner, education, ever worked outside Vietnam, age of first sex, 100\% condom use with clients, past history of STIs, direct sex work, positive syphilis serology and NG/CT.

$\mathrm{Cl}$, confidence interval; NG/CT, Neisseria gonorrhoeae and/or Chlamydia trachomatis. 
Although injecting drug use (IDU) is an important mode of HIV transmission in Vietnam, only 4\% of FSWs in our overall study population had used opiates ${ }^{6}$ compared with $13 \%$ in Ho Chi Minh where $16 \%$ were HIV positive. ${ }^{9}$ While significant rates of opiate use in FSWs were found in Lai Chau (6\%) and Kien Giang (9\%), ${ }^{6}$ our study found low HIV prevalences in FSWs in these provinces. Clearly, sex work and opiate use vary throughout the country but the potential for further spread of HIV appears to be high, given current patterns of sexual mixing patterns and low levels of condom use. ${ }^{10}$

FSWs with a low income and $\geqslant 9$ clients per week were at increased risk of HIV. These variables were also associated with a higher rate of chlamydia/gonorrhoea in the same population of FSW that had a cohabiting partner ${ }^{6}$ and may reflect poverty and the financial need to sell sex to many low paying customers. These FSWs may not be able to access STI and HIV prevention programmes easily because of pressures of work. Clearly, mobile clinics and outreach programmes are likely to offer these women a more user friendly service than the traditional STI clinic model.

FSWs with earlier sexual debut were also at increased risk of HIV. Commencing sex work at an early age has been associated with HIV in Thailand and may reflect poor awareness of safer sex practices. ${ }^{11}$ It may also reflect the vulnerability of the immature cervix to HIV despite limited numbers of sexual partners. ${ }^{12}$

The borderline association with syphilis should serve as a reminder that this condition is prevalent in Vietnam with Kien Giang having higher levels than the other four provinces. ${ }^{6}$

Having a non-paying regular partner as a protective factor for HIV could reflect fewer opportunities to have sex with other clients or partners. The high levels of HIV reported previously in FSWs in An Giang ${ }^{7}$ reinforce the need for health agencies to adapt rapidly to local changes in HIV epidemiology. Our data suggest this may have started to happen and offers some optimism for the future.

\section{ACKNOWLEDGEMENTS}

We wish to thank all staff involved in the study in the five provinces particularly those in the Preventive Medicine Centres (Dr Phung Xuan Ty, Dr Le Cong Sy, Dr Mai Hoang Anh, Dr Nguyen Ngoc An, Dr Le Van Xanh), Indu Bhushan and Peter Godwin of the Asian Development Bank, Dr Trinh Quan Huan, Dr Nguyen Thanh Long, AIDS Division, Ministry of Health and Ms Tran Thi Thanh Mai, Commission for Population, Family and Children, Population and Family Health, Hanoi for their help and support in undertaking this study. Funding was through the Japanese Fund for Poverty
Reduction, Community Action for Preventing HIV/AIDS- 9006 grant administered by the Asian Development Bank.

\section{CONTRIBUTORS}

Conception and design, NT, NO'F; survey training, NT, VN, LT; data collection and field survey monitoring: $\mathrm{NT}, \mathrm{VN}$; analysis and interpretation of data NT, VN, KN, NO'F; laboratory testing LT; critical revision of article NT, NO'F.

\section{Authors' affiliations}

N V Thuong, V T Nhung, K V Nghia, L T Tram, Pasteur Institute, Ho Chi Minh City, Vietnam

N O'Farrell, Pasteur Suite, Ealing Hospital and London School of Hygiene and Tropical Medicine, London, UK

Correspondence to: Dr Nigel O'Farrell, Pasteur Suite, Ealing Hospital, London UB1 3HW, UK; ofarrell@postmaster.co.uk

Accepted for publication 15 April 2005

\section{REFERENCES}

1 Hien NT, Long NT, Huan TQ. HIV/AIDS epidemics in Vietnam: evolution and responses. AIDS Education and Prevention 2004; 16(Suppl A):137-54.

2 Rekart ML. Sex in the city: sexual behaviour, societal change and STDs in Saigon. Sex Trans Inf 2002;78(Suppl 1):i47-i54.

3 www.jfpr-hiv.org

4 Tuan NA, Thang BD, Hien NT, et al. Baseline survey report in 5 provincescommunity action for preventing HIV/AIDS Project in Lai Chau, Quang Tri, Dong Thap, An Giang and Kien Giang provinces 2002.

$5 \mathrm{STI} / \mathrm{HIV}$ survey among female sex workers in 5 border provinces, Vietnam 2002. Project Community Action for preventing HIV/AIDS (JFPR: Reg 9006). Hanoi: Medical Publishing House, 2003. (www.jfpr-hiv.org/stihiv_survey_sw_vn.pdf).

6 Thuong NV, Long NT, Hung ND, et al. Sexually transmitted infections in female sex workers in five border provinces of Vietnam. Sex Transm Dis 2005;32:550-6.

7 O'Farrell N, Anh MH, Thuong NV, et al. Variation in HIV prevalence in female sex workers in An Giang, Vietnam: what is a representative sample? XV International Conference on AIDS, Bangkok, Thailand, 11-16 July 2004, Abstract WePeC6186.

8 NCHADS. Low prevalence of sexually transmitted infections in Cambodia supports recent behavioural and HIV seroprevalence trends, 2001. Cambodia: STI Prevalence Survey, February, 2002.

9 Tuan NA, Hien NT, Chi PK, et al. Intravenous drug use among street-based sex workers. Sex Transm Dis 2004;31:15-19.

10 Tuan NA, Fylkesnes K, Thang BD, et al. HIV infection patterns and risk behaviours in different population groups and provinces in Vietnam: close links between drug injection related and heterosexual epidemics. XV International Conference on AIDS, Bangkok, Thailand, 11-16 July 2004, Abstract MoPeC3449.

11 Limpakarnjanarat K, Mastro T, Saisorn S, et al. HIV-1 and other sexually transmitted infections in female sex workers in a cohort of female sex workers in Chiang Rai, Thailand. Sex Trans Infect 1999;75:30-5.

12 O'Farrell N, Windsor I. Sexual behaviour in HIV-1 seropositive Zulu men and women in Durban, South Africa. J AIDS 1991;4:1258-9. 